\title{
Bacterial pathogens in conjunctivitis and corneal ulcers with special reference to Streptococcus pneumoniae, in early 2008, in the National Eye Hospital, Sri Lanka
}

\author{
DLB Piyasiri ${ }^{1}$, P Chandrasiri $^{2}$, PR Wijesinghe ${ }^{3}$ \\ Sri Lankan Journal of Infectious Diseases 2020 Vol.10(2):125-133 \\ DOI: http://dx.doi.org/10.4038/sljid.v10i2.8288
}

\begin{abstract}
Introduction: The most important and frequently occurring eye infections are conjunctivitis and corneal ulcers. Though the significance of Streptococcus pneumoniae in eye infections is wellknown, identification of causative serotypes is important for comparison with serotypes causing invasive diseases.
\end{abstract}

Objectives: To describe the spectrum and characteristics of bacterial pathogens associated with conjunctivitis and corneal ulcers with special reference to $S$. pneumoniae.

Methods: A descriptive cross-sectional study was carried out from January to April 2008 in which samples were collected from patients above one month old with suspected conjunctivitis or corneal ulcers seen in the out-patients department and wards of the National Eye Hospital, Colombo, Sri Lanka. Both eye swabs $(n=322)$ and corneal buttons $(n=31)$ were cultured. Isolated bacteriawere identified as far as possible and antibiotic sensitivity testing done. S. pneumoniae isolates were stored in sheep blood agar slants at $-70^{\circ} \mathrm{C}$ and serotyped.

Results: There were 296 conjunctival swabs and 26 corneal swabs. From 296 conjunctival swabs 118 (39.8\%) samples yielded no bacterial growth. There were 185 bacterial isolates from 159 significant positive samples. The most frequently isolated bacteria were coagulase negative Staphylococcus sp. (n=58: 31.4\%), and second commonest was $S$. pneumoniae (n=33: 17.84\%). From 57 samples from patients with corneal ulcers, 47 (82\%) did not yield any bacterial growth.

Sensitivity to chloramphenicol was high among Staphylococcus sp., S. pneumoniae, Haemophilus influenzae, Moraxella sp and coliforms. Among pneumococcal isolates serotyped, 19F was predominant.

${ }^{1}$ National Eye Hospital, Colombo, Sri Lanka

${ }^{2}$ National Hospital Sri Lanka, Colombo, Sri Lanka

${ }^{3}$ Epidemiology unit, Colombo, Sri Lanka

Address for correspondence: Dr Bhagya Piyasiri, National Eye Hospital, Colombo, Sri Lanka.

Tel: +94777654317 E-mail: bhagyapiyasiri@yahoo.co.uk@ iDttps://orcid.org/0000-0002-4660-311X

Received 1 April 2020 and revised version accepted 27June 2020

(c) (†)

This an open-access article distributed under the terms of the Creative Commons Attribution License, which permits unrestricted use, distribution, and reproduction in any medium, provided the original author and source are credited. 
Conclusion: Coagulase negative staphylococci were predominantly isolated from eye swabs and corneal buttons, but the most significant frequent isolate was $S$. pneumoniae. Except Pseudomonas, $>90 \%$ isolates were sensitive to chloramphenicol. The predominant pneumococcal serotype was $19 \mathrm{~F}$.

Keywords: bacterial pathogens, conjunctivitis, corneal ulcers, Streptococcus pneumoniae

\section{Introduction}

Two of the most important and frequently occurring eye infections, irrespective of age are conjunctivitis and corneal ulcers. Though conjunctivitis could be very mild, outbreaks are known to occur and it is a nuisance to affected patients, with pain, irritation and eye discharge accompanied by social stigmata. ${ }^{1}$ Most frequently, conjunctivitis is caused by viruses or bacteria including sexually transmitted diseases. ${ }^{1}$ Acute viral infections can also be secondarily infected with bacteria in chronic inflammation. Both conjunctivitis and corneal ulcers occur with bacterial pathogens like Staphylococcus sp., pneumococci, and Haemophilus sp. Parasites and fungi may also play a part. External eye infections become chronic if left untreated or partially treated and may lead to blindness. Conjunctivitis is usually self-limiting though topical antibiotics play a major role in treatment if the causative organism is identified. ${ }^{2} \mathrm{~A}$ few studies have been done on ocular infections in Sri Lanka, although most have been analyzed without grouping into type of infection or by the age group. ${ }^{3-6}$

Though the significance of $S$. pneumoniae in eye infections is well-known, ${ }^{2,3,5}$ a detailed analysis with sero-grouping is still important to compare with serotypes associated with invasive pneumococcal diseases. This data will be valuable in the decision of introducing a pneumococcal vaccine in the national immunization programme in Sri Lanka.

The objectives of the current study were to describe the spectrum and characteristics of bacterial pathogens associated with conjunctivitis and corneal ulcers with special reference to $S$. pneumoniae.

\section{Methods}

A descriptive cross-sectional study was carried out from January to April 2008 in the National Eye Hospital, Colombo, Sri Lanka. Ethical approval was obtained from the ERC, Faculty of Medicine, Colombo. The study population included both in-ward and the out-patient department (OPD) patients with suspected conjunctivitis and corneal ulcers. Patients above one month old were included while patients less than one month were excluded as they can have sexually transmitted eye infections ${ }^{1}$ which were not tested within the study scope. Eye swabs were also not collected from patients already receiving topical or systemic antibiotics, but corneal buttons were accepted. The total sample size was calculated as 322 according to the culture positivity rate $(70 \%)$ of the study done by Agarwala et al (1967) using the standard formula $n=Z^{2} p q / d^{2}$ where $Z$ is the value for $95 \%$ confidence level (1.96), $p$ is the prevalence of culture positivity (0.7) and $q$ is (1-p) with $\mathrm{d}$ being the margin of error $(5 \%){ }^{7}$ 
Eye swabs were collected from the patients following explanation of the procedure and obtaining informed written consent. Whenever possible, swabs were collected in duplicate for direct Gram stain and for culture from patients seen in the OPD. In-ward samples were mainly from patients with corneal ulcers, often as a single swab. Corneal buttons sent from patients with suspected keratitis were also included in the study. Samples were collected only during day time and were plated within 2 hours of collection on 3 media; sheep-blood agar, chocolate agar and MacConkey agar. All plates were incubated at $37^{\circ} \mathrm{C}$ for $18-24$ hours and in-addition, blood and chocolate plates were kept in the candle jar $\left(5 \% \mathrm{CO}_{2}\right)$ within the incubator. Direct Gram stain was performed only for the swabs received in duplicate.

Bacteria were identified as far as possible with available biochemical tests such as tube coagulase test for Staphylococcus sp, optochin sensitivity (>14mm zone) and bile solubility to identify $S$. pneumoniae, and oxidase test and Kligler iron agar to differentiate between Pseudomonas sp and coliform sp. Gram stain appearance of Gram negative cocci including intracellular forms along with colony morphology and oxidase test were used for presumptive identification of Moraxella sp. while " $\mathrm{X}$ " and "V" factors were used to confirm the identity of $H$. influenzae.

Antibiotic sensitivity testing (ABST) was done according to the CLSI 2008 standards. ${ }^{9}$ The minimum inhibitory concentration (MIC) of penicillin for S. pneumoniae was done using Oxoid M.I.C.Evaluator ${ }^{\mathrm{TM}}$ (M.I.C.E.) strips. The MIC of cefotaxime for S. pneumoniae could not be tested due to lack of funding. Oxacillin resistance was determined by a reduced zone diameter $(<19 \mathrm{~mm})$ or with no zone around the disc. Inducible clindamycin resistance was checked in both staphylococci and streptococci by the erythromycin and clindamycin disc approximation test. ${ }^{9}$ Stokes ABST method was performed for Moraxella spp and for sensitivity of Staphylococcus sp. To fusidic acid.

All isolates of $S$. pneumoniae were stored in sheep blood agar slants at $-70^{\circ} \mathrm{C}$ and later sent to Christian Medical College, Vellore, India for sero-grouping by a co-agglutination method.

\section{Results}

A total 322 eye swabs and 31 corneal buttons were collected and processed during the study period.

\section{Direct Gram stain of swabs}

Of the 203 swabs which had a direct Gram stain performed, 46(22.7\%) were smear positive with organisms and pus cells and 41 were culture positive for the organisms predicted according to the Gram stain morphology.

Eighty one smears $(39.9 \%)$ had pus cells without organisms, 31 of which were culture positive. Seventy six smears (37.4\%) were negative for both organisms and pus cells of which 36 were culture positive. There was a significant association between direct Gram stain with organisms and positive cultures (Fisher's exact test, $\mathrm{p}<0.0001$ ).

\section{Positive cultures}

Of the 296 swabs from patients with conjunctivitis, there was no bacterial growth in 118 samples $(39.8 \%)$ while 135 samples yielded a single isolate $(45.6 \%)$ and 24 samples yielded 2 or more 
isolates $(8.1 \%)$. Nineteen samples $(6.4 \%)$ yielded isolates which were considered as contaminants and non-pathogenic (micrococci and diphtheroids) while the remaining isolates were considered significant, especially if there was a heavy pure growth.

Table 1: Bacteria isolated from patients with conjunctivitis $(\mathbf{n}=\mathbf{1 8 5})$

\begin{tabular}{|l|c|c|}
\hline Organism & n & $\%$ \\
\hline $\begin{array}{l}\text { Coagulase negative } \\
\text { Staphylococcus (CoNS) }\end{array}$ & 58 & 31.4 \\
\hline Streptococcus pneumoniae & 33 & 17.8 \\
\hline Haemophilus influenzae & 29 & 15.7 \\
\hline Viridans streptococcus & 19 & 10.3 \\
\hline Staphylococcus aureus & 15 & 8 \\
\hline Coliform sp. & 13 & 7 \\
\hline Moraxella sp. & 12 & 6.5 \\
\hline Pseudomonas sp. & 4 & 2.2 \\
\hline Streptococcus pyogenes & 2 & 1.1 \\
\hline Total & 185 & 100 \\
\hline
\end{tabular}

There were 185 bacterial isolates from a total of 159 significant positive samples from patients with conjunctivitis. The isolated organisms are shown in Table 1.

Of the 26 swabs from patients with corneal ulcers, $18(69.2 \%)$ did not yield any bacterial growth while 29 of 31 corneal buttons $(93.5 \%)$ were negative (Table 2).

Table 2: Bacteria isolated from patients with corneal ulcers $(n=8)$

\begin{tabular}{|l|c|l|l|l|l|}
\multicolumn{3}{c|}{ Corneal swabs } & \multicolumn{3}{c}{ Corneal buttons } \\
\hline Organism & $\mathrm{n}$ & $\%$ & Organism & $\mathrm{n}$ & $\%$ \\
\hline CoNS & 4 & $50 \%$ & Viridans streptococcus & 1 & 50 \\
\hline Pseudomonas sp. & 2 & $25 \%$ & Aerobic spore bearer & 1 & 50 \\
\hline Viridans streptococcus & 2 & $25 \%$ & & & \\
\hline Total & 8 & $100 \%$ & Total & 2 & 100 \\
\hline
\end{tabular}

\section{Antibiotic sensitivity}

While all CoNS and $S$. aureus were sensitive to vancomycin, the sensitivity to chloramphenicol and fusidic acid was $64 \%$ and $62 \%$ respectively. There were only 3 methicillin resistant $S$. aureus (MRSA). Sensitivity to chloramphenicol among $H$. influenzae and Moraxella sp. was $93 \%$ and 91\% respectively. All 6 isolates of Pseudomonas sp. were sensitive to ciprofloxacin, gentamicin, netilmicin and ceftazidime. The sensitivity to amikacin was $83 \%$. Of the 13 coliforms, sensitivity to gentamicin, ciprofloxacin and chloramphenicol was $64 \%, 88 \%$ and $86 \%$ respectively. Resistance to co-amoxiclav was $64 \%$. 
Table 3 - Pathogens in relation to age of patients

\begin{tabular}{|c|c|c|c|c|c|c|c|c|c|}
\hline 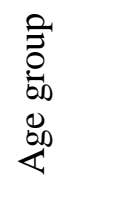 & $\begin{array}{l}\tilde{z} \\
0 \\
0\end{array}$ & 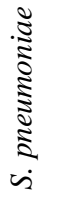 & 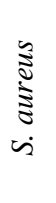 & 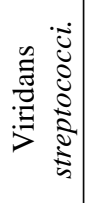 & 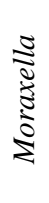 & 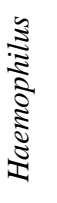 & 营 के & 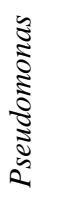 & $\begin{array}{l}\tilde{y} \\
\tilde{\Xi} \\
\infty \\
\vdots \\
\dot{0} \\
\dot{s}\end{array}$ \\
\hline $1 \mathrm{~m}-1 \mathrm{yr}$ & 38 & 21 & 10 & 14 & 7 & 13 & 8 & 3 & 2 \\
\hline $1-5$ yrs & 13 & 5 & 2 & 4 & 5 & 15 & - & - & - \\
\hline$>5$ yrs & 1 & 3 & - & - & - & - & 2 & - & - \\
\hline$>21 y$ & 2 & 4 & 2 & 1 & - & - & 3 & 1 & - \\
\hline$>60 \mathrm{yrs}$ & 4 & - & 1 & 0 & - & 1 & - & - & - \\
\hline
\end{tabular}

Data were analyzed in relation to 5 age groups (Table 3). All pathogens except $H$. influenzae were seen most frequently among children aged 1 month to 1 year. H. influenzae was seen commonly among the age group 1 to 5 years.

S. pneumoniae was seen also among the adult age groups.

Table 4: Pathogens in relation to the duration of the symptoms in patients with conjunctivitis.

\begin{tabular}{|c|c|c|c|c|}
\hline Pathogen & 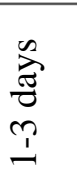 & $\frac{\frac{y}{3}}{\frac{1}{2}}$ & $\frac{\dot{v}}{\underline{\Xi}}$ & $\begin{array}{l}\bar{\Xi} \\
\overline{0} \\
\text { त }\end{array}$ \\
\hline Polymicrobial & 4 & 4 & 4 & 12 \\
\hline S. pneumoniae & 3 & 1 & 1 & 28 \\
\hline H. influenzae & 11 & 7 & 5 & 6 \\
\hline Moraxella sp. & 0 & 3 & 2 & 7 \\
\hline CoNS & 4 & 10 & 21 & 23 \\
\hline S. aureus & 0 & 2 & 4 & 9 \\
\hline S.pyogenes & 0 & 0 & 0 & 2 \\
\hline Viridans streptococci. & 3 & 4 & 3 & 9 \\
\hline Pseudomonas sp. & 0 & 0 & 0 & 4 \\
\hline Coliforms & 0 & 0 & 8 & 5 \\
\hline
\end{tabular}

H. influenzae was seen most frequently in relation to acute illness, with a history of symptoms for 1 to 3 days and up to 1 week. In contrast, symptoms for more than one month were noted with polymicrobial infections,

S. pneumoniae, Moraxella sp, and $S$. aureus. S. pyogenes and

Pseudomonas sp. were only seen in patients with a history of more than one month. CoNS and viridans streptococcus were seen in both acute and chronic illnesses (Table 4).

\section{Streptococcus pneumoniae}

There were 33 strains of $S$. pneumoniae isolated during the 4 month period of the study, $9(27.3 \%)$ of which were recovered from polymicrobial cultures. Of the polymicrobial cultures, $5(55.6 \%)$ included $H$. influenzae while the remaining 4 included CoNS $(\mathrm{n}=2)$, Moraxella $\mathrm{sp} .(\mathrm{n}=1)$, and coliforms $(\mathrm{n}=1)$.

Patients aged 1 month to 1 year were the most affected by $S$. pneumoniae (21/33) and had prolonged duration of the disease (28/33). Five patients with chronic conjunctivitis had possible naso-lacrimal duct obstruction.

Twenty three strains $(69.7 \%)$ of S. pneumoniae were resistant to oxacillin and MIC of penicillin. of the 10 sensitive strains was $0.016 \mu \mathrm{g} / \mathrm{mL}$. Of the oxacillin resistant strains, three had a MIC of more than $1 \mu \mathrm{g} / \mathrm{mL}$. None were resistant according to the MIC breakpoint for parenteral penicillin $(<2 \mu \mathrm{g} / \mathrm{mL})$ for non-meningeal pneumococcal isolates (CLSI January 2008). ${ }^{9}$ 
Sensitivity to erythromycin was $61.5 \%$ and sensitivity to clindamycin $84.6 \%$. No inducible clindamycin resistance was detected. There was $96.9 \%$ sensitivity for chloramphenicol while all (100\%) were sensitive to vancomycin.

\section{Serogrouping of S. pneumoniae}

Only 31 isolates were sent to Christian Medical College, Vellore, India, to be serogrouped and serotyping was attempted on 23 isolates. Of the 31,5 had lost viability during transport and another 3 were contaminated and not available for serotyping. The predominant serotype was $19 \mathrm{~F}$ (7/23, $30 \%$ ) while 6 isolates were $23 \mathrm{~A}$, and one isolate each were type 16A, 3, 19B, 33B, 22A, 15C, and 9A. Three were non-typeable. While 5 of the patients with 19F, 5 of the ones with $23 \mathrm{~A}$, and 4 of the patients with other serotypes were infants, there was one 11 year old with $19 \mathrm{~F}$ and four 1-2 year old children with 23A, 19B, 22A and non-typeable serotypes respectively. The rest were adults.

\section{Discussion}

Conjunctivitis and corneal ulcers are the most common eye infections affecting people in the community. The bacterial spectrum causing external eye infections may vary from time to time for a variety of reasons, usually multi-factorial, including the geographical location and urbanization level of the studied population. ${ }^{10}$ There can also be outbreaks of conjunctivitis or kerato conjunctivitis in the presence of overcrowding and congestion as in schools, universities and military camps ${ }^{11}$. It is therefore important to have accurate information of the epidemiology and microbiology of those infections for appropriate management of patients.

Direct smears which had organisms and pus cells yielded a high rate of positive culture (41 of 46 smears) for the predicted organisms. Only 5 were negative which may be due to unknown prior antibiotic therapy. There was a significant association between direct Gram stain with organisms and positive cultures $(\mathrm{p}<0.0001)$.

In this study, eye swabs taken from patients with conjunctivitis and corneal ulcers yielded 57.8\% culture positivity with 197 apparently significant isolates including CoNS (32\%), S. pneumoniae (17\%), H. influenzae (15\%), viridans streptococci (10.9\%), and S. aureus (7.8\%). Agarwala et al obtained a higher positivity at $70 \%$ with CoNS $(67.5 \%)$, S. aureus $(10 \%)$, and a much lower isolation of S. pneumoniae (1.5\%). In contrast, H. influenzae (44\%) followed by S. pneumoniae (30\%) were found in the study by Martinez et al among paediatric patients. ${ }^{2}$ Further, similar to the $8.1 \%$ polymicrobial cultures including the combination of $H$. influenzae and $S$. pneumoniae in $21 \%$ of those in the current study, Martinez et $\mathrm{al}^{2}$ also reported $8.1 \%$ of mixed cultures with the same two organisms. Co-existence of S. pneumoniae and $H$. influenzae in the mixed infections is probably because $S$. pneumoniae produces V factor by alpha haemolysis of sheep erythrocytes on blood agar which is utilized by $H$. influenzae. ${ }^{12}$

CoNS was the most frequently isolated pathogen $(58,31 \%)$. However, contamination during difficult swabbing cannot be excluded, especially in very small children as obtaining a proper conjunctival swab was extremely difficult. 
In patients with $H$. influenzae, the history with acute onset and short duration was typical. Eighteen of 29 isolates were from patients who had a history of <1 week duration. These patients usually did not have any predisposing factors. Also $H$. influenzae was the commonest isolate in the 1-5 year age group in contrast to the findings of Martinez et al. in (2004) in which S. pneumoniae was the commonest in the 2-6 year age group. ${ }^{2}$ In the current study, 5 patients with $S$. pneumoniae had possible naso-lacrimal blockage which resulted in chronic conjunctivitis which is well described in the study of Mahajan et al. (1987). ${ }^{13}$

Sensitivity to chloramphenicol among $S$.pneumoniae, coliforms, viridians streptococcus, $H$. influenzae and Moraxella sp. was above $85 \%$ while it was $64 \%$ among Staphylococcus sp. Mahajan et al (1987) ${ }^{13}$ reported 69\% sensitivity for $S$. pneumoniae while Martinez et al (2004) ${ }^{2}$ observed high chloramphenicol sensitivity among several pathogens. Chloramphenicol is a frequently used empirical ophthalmic treatment. Its use is justified by these observations.

According to CLSI breakpoint of $0.06 \mu \mathrm{g} / \mathrm{mL}$ (before 2008), 3(9.1\%) of our isolates were resistant to penicillin $(>1 \mu \mathrm{g} / \mathrm{mL})$. $20(60.6 \%)$ were intermediate $(0.12-1 \mu \mathrm{g} / \mathrm{mL})$ and $10(30.3 \%)$ were sensitive which tallied with the results of oxacillin disc sensitivity tests. However, a major change in penicillin MIC breakpoints for parenteral non-meningeal pneumococcal isolates was introduced by CLSI in January 2008, where MICs to indicate penicillin sensitivity was raised to $2 \mu \mathrm{g} / \mathrm{mL}$. By the revised MIC, all pneumococcal isolates in the current study were penicillin sensitive. ${ }^{9}$

Although there are 3 pneumococcal conjugate vaccines in the world, none are included in the national immunization schedule for children in Sri Lanka to date. The present study isolated 2 pneumococcal tridecavalent conjugate vaccine serotypes, $19 \mathrm{~F}$ and $3(34.8 \%)$, and 3 heptavalentvaccine (PCV7)-related serotypes; 9A, 19B, and 23A. ${ }^{14}$ PCV7-related serotypes are those within the same serogroup as the PCV7 serotypes that were either assumed or known to be cross-reactive with PCV7 serotypes. ${ }^{14}$ In our study, both vaccine and vaccine-related serotypes together make $69.6 \%$ of total serotypes. Both heptavalent vaccine and the 10 -valent vaccine have the coverage for $19 \mathrm{~F}$ serotype and presumptive coverage for related serotypes.

Most patients from whom pneumococcal 19F were isolated were aged 2-10 months $(\mathrm{n}=5)$ and 4 such children had purulent eye discharge since birth. Another patient with serotype 19F was 11 years old, had naso-lacrimal duct obstruction and was on frequent probing for dilatation. The results of the present study raise the question of whether pneumococcal conjugate vaccine should be recommended for children, especially those with risk factors for chronic conjunctivitis such as naso-lacrimal duct obstruction.

The current study is the first Sri Lankan study with detailed analysis of ocular strains of $S$. pneumoniae including serotyping of these strains. Kulkarni et al described serotypes of $S$. pneumoniae in invasive disease in Sri Lanka in 2008. They found 19F, 14, 6B, and 23F (15\% each) were most common followed by serotypes $29,3,20,15 \mathrm{~B}$, and 16 (5\% each). ${ }^{15}$ Wijesinghe et al (2008) described a similar phenomenon concluding that 19F, 23F and 6B (13\% each) were the commonest serotypes causing invasive pneumococcal diseases in Sri Lanka. ${ }^{16}$ This data shows interesting similarity between the serotypes causing invasive diseases and the serotypes causing non-invasive eye infections like conjunctivitis and corneal ulcers in Sri Lanka. 


\section{Conclusion}

Coagulase negative Staphylococcus spp was the commonest isolate from eye swabs from patients with conjunctivitis and corneal ulcers. As the possibility of contamination with skin flora cannot be excluded, S. pneumoniae can be considered the most significant and most frequent pathogen isolated. Positive direct smears are valuable in treating patients empirically before culture results are available. All isolated pneumococci were sensitive to penicillin (MIC $<2 \mu \mathrm{g} / \mathrm{mL}$ ) while MRSA prevalence was $20 \%$. As $>90 \%$ of all isolates were sensitive to chloramphenicol (except Pseudomonas spp), it can be used in empirical treatment. Of pneumococcal serotypes causing external eye infections, $70 \%$ were conjugate vaccine constituents or vaccine-related serotypes, suggesting the possibility of vaccination as a means of reducing infection in children with risk factors such as naso-lacrimal duct obstruction.

Acknowledgement: The authors acknowledge the funding by the Epidemiology Unit of Sri Lanka, and the support given by the clinical and laboratory staff of the National Eye Hospital, Colombo, Christian Medical College, Vellore, Medical Research Institute, Borella, and National Hospital of Sri Lanka, Colombo, with gratitude.

Conflicts of Interest: The authors declare no conflicts of interest at the time of the research.

\section{References}

1. Morrow G.L., Abbot R.C., Conjunctivitis, Am Fam Physician 1998;57(4):735-746 No doi

2. Martinez B.O., Ruiz R.M., Perez R.M., Bacterial conjunctivitis: Most prevalent pathogens and their antibiotic sensitivity, An Pediatr (Barc) 2004; 61(1):32-6 doi: 10.1016/s1695-4033(04)78350-2

3. Perera C., Guzek J., Microbiological study of eye infections in Galle District, SL, Bulletin of Sri Lanka College of Microbiologists 1991: OP4 No doi

4. Seneviratne R., Chandrasiri P., Dias J., Infectious agents of corneal ulcers, endophthalmitis, \& other severe eye Infections; Bulletin of Sri Lanka College of Microbiologists2004; 2(1): OP8 No doi

5. Karunanayake L., Senanayake S., Microbiological profile of conjunctival \& corneal eye infections in T. H. Kandy, Bulletin of Sri Lanka College of Microbiologists 2006; 4(1):21-22 No doi

6. Edussuriya L., Chandrasiri P., Samarasekara A., et al, Etiological agents of ophthalmic infections, Journal of the College of Ophthalmologists in Sri Lanka 2007; 13(1):56-57 No doi

7. Agarwala H.S., Raichaudhury M., Munsi N.K., Bacterial flora in acute conjunctivitis, Indian $J$ Ophthalmol 1967; 15(2):58-66 No doi

8. Barrow GI, Feltham RKA (eds), Cowan and Steel's Manual for the identification of medical bacteria (3rd ed), Press Syndicate of the University of Cambridge, 1993

9. Performance Standards for Antimicrobial Susceptibility Testing; Eighteenth Informational Supplement, Wayne, Pennsylvania, USA, CLSI, 2008

10. Pantoja-Melendez C.A., Bautista-de Lucio V.M., Ortiz-Casas M., et al, Eye coinfections. In: Advances in Common Eye Infections, Intech Open, doi: http://dx.doi.org/10.5772/64702

11. Martin M., Turco J.H., Zegans M.E., et al. An outbreak of conjunctivitis due to atypical Streptococcus pneumoniae, $N$ Engl J Med 2003; 348(12):1112-1121

doi: http://dx.doi.org/10.1056/NEJMoa022521

12. Elliott J.A., Facklam R.R., Nathan C., et al, Co-isolation of Streptococcus pneumoniae and Haemophilus influenzae from middle ear fluid and sputum: Effect on MIC results, J Clin Microbiol 1999; 37(1):277 doi: https://doi.org/10.1128/JCM.37.1.277-277.1999

13. Mahajan V.M., Bareja U., Prakash K., Pneumococci in ocular diseases of children and their 
treatment, Ann Tropical Paediatrics. 1987; 7(4):270-3

doi: http://dx.doi.org/10.1080/02724936.1987.11748523

14. Hsu H.E., Shutt K.A., Moore M.R., et al, Effect of pneumococcal conjugate vaccine on pneumococcal meningitis, $N$ Engl J Med. 2009; 360(3):244-256

doi: http://dx.doi.org/10.1056/NEJMoa0800836

15. Kulkarni P., Thomas K., Steinhoff M., et al, Epidemiology of invasive pneumococcal disease in Nepal, Sri Lanka and India : An update on three years of SAPNA surveillance (Nov 2004-Dec 2007), ISPPD-6 abstract book, 2008: 95 doi: 10.13140/RG.2.1.3198.3841

16. Wijesinghe P., Karunarathna K., Abeysinghe N., et al, Surveillance of pneumococcal invasive diseases in Sri Lanka under South Asian pneumococcal network alliance (SAPNA), ISPPD-6 abstract book. 2008:131-132 doi: 10.13140/RG.2.1.4449.7126 